\title{
Correction to: Hospital-acquired malnutrition in paediatric patients: a multicenter trial focusing on prevalence, risk factors, and impact on clinical outcomes
}

\author{
Suchaorn Saengnipanthkul ${ }^{1,2}$ (1) Nalinee Chongviriyaphan ${ }^{2,3} \cdot$ Narumon Densupsoontorn $^{2,4}$. \\ Amnuayporn Apiraksakorn ${ }^{5}$. Jitjira Chaiyarit ${ }^{6}$. Supawan Kunnangja ${ }^{4}$. Sasiwimol Wongpratoom ${ }^{1}$. \\ Supawan Papakhee ${ }^{1}$. Wipada Det-amnatkul ${ }^{5}$. Jittima Monwiratkul ${ }^{3}$. Puthita Saengpanit ${ }^{4}$. \\ Pajaree Limthongthang ${ }^{2}$. Charnchai Panthongviriyakul ${ }^{1,2}$
}

Published online: 28 October 2021

c) Springer-Verlag GmbH Germany, part of Springer Nature 2021

\section{Correction to: \\ European Journal of Pediatrics (2021) 180:1761-1767 https://doi.org/10.1007/s00431-021-03957-9}

In the original published version of this article, the uploaded Supplementary Material 1, 2, and 3 were incorrect. The correct materials have been uploaded and replace the incorrect materials.

The original article has been corrected.
Publisher's Note Springer Nature remains neutral with regard to jurisdictional claims in published maps and institutional affiliations.

The original article can be found online at https://doi.org/10.1007/ s00431-021-03957-9.

Suchaorn Saengnipanthkul

suchsa@kku.ac.th; suchaorn@hotmail.com

1 Division of Nutrition, Department of Pediatrics, Faculty of Medicine Srinagarind Hospital, Khon Kaen University, Khon Kaen 40002, Thailand

2 Pediatric Nutrition Association of Thailand, PedNAT, Bangkok, Thailand

3 Division of Nutrition, Department of Pediatrics, Faculty of Medicine Ramathibodi Hospital, Mahidol University, Bangkok, Thailand

4 Division of Nutrition, Department of Pediatrics, Faculty of Medicine Siriraj Hospital, Mahidol University, Bangkok, Thailand

5 Department of Pediatrics, Khon Kaen Hospital, Khon Kaen, Thailand

6 Clinical Epidemiology Unit, Khon Kaen University, Khon Kaen, Thailand 